\title{
Effects of thermal environment on sleep and circadian rhythm
}

\author{
Kazue Okamoto-Mizuno ${ }^{1 *}$ and Koh Mizuno²
}

\begin{abstract}
The thermal environment is one of the most important factors that can affect human sleep. The stereotypical effects of heat or cold exposure are increased wakefulness and decreased rapid eye movement sleep and slow wave sleep. These effects of the thermal environment on sleep stages are strongly linked to thermoregulation, which affects the mechanism regulating sleep. The effects on sleep stages also differ depending on the use of bedding and/or clothing. In semi-nude subjects, sleep stages are more affected by cold exposure than heat exposure. In real-life situations where bedding and clothing are used, heat exposure increases wakefulness and decreases slow wave sleep and rapid eye movement sleep. Humid heat exposure further increases thermal load during sleep and affects sleep stages and thermoregulation. On the other hand, cold exposure does not affect sleep stages, though the use of beddings and clothing during sleep is critical in supporting thermoregulation and sleep in cold exposure. However, cold exposure affects cardiac autonomic response during sleep without affecting sleep stages and subjective sensations. These results indicate that the impact of cold exposure may be greater than that of heat exposure in real-life situations; thus, further studies are warranted that consider the effect of cold exposure on sleep and other physiological parameters.
\end{abstract}

Keywords: Cold, Heat, Sleep, Thermal environment, Thermoregulation

\section{Review}

The thermal environment is a key determinant of sleep because thermoregulation is strongly linked to the mechanism regulating sleep [1]. Excessively high or low ambient temperature (Ta) may affect sleep even in healthy humans without insomnia. Furthermore, disturbed nocturnal sleep affects not only daytime activities, but is also related to various adverse health effects, such as obesity [2], quality of life, and even mortality [3,4]. These findings indicate that maintaining a comfortable thermal sleep environment is important for sleep maintenance as well as daytime activities and health status. Among the various thermal environmental factors, the relationship between $\mathrm{Ta}$ and the use of clothing and beddings differs greatly between humans and animals. The effects of Ta on sleep stages differ depending on whether subjects are semi-nude or use bedding and clothing, making it difficult to extrapolate the results of animal studies

\footnotetext{
* Correspondence: kazue@tfu-mail.tfu.ac.jp

${ }^{1}$ Kansei Fukushi Research Center, Tohoku Fukushi University, 1-149-6

Kunimigaoka Aoba Sendai, Miyagi, 981-0935, Japan

Full list of author information is available at the end of the article
}

related to thermal environment and sleep to humans. The use of clothing and beddings greatly aid in maintaining the body temperature at an acceptable thermal state in a variety of environments by providing thermal resistance for the human body from its environment [5]. In this review, based on our studies related to thermoregulation and sleep in humans, the effects of the thermal environment on sleep and circadian rhythm are discussed. The effects of heat and cold exposure, including bedding and clothing conditions along with their effects in the elderly, are of special interest, because these are the important factors that most impact sleep and thermoregulation.

\section{Sleep and thermoregulation}

Many previous studies in humans indicate that sleep is strongly linked to thermoregulation (For examples, see $[1,6])$, which is primarily controlled by circadian rhythm and sleep regulation. Humans have a sleep-wake rhythm that is repeated in a 24-hour cycle. The core body temperature (Tcore), which also cycles along with the sleep-wake rhythm, decreases during the nocturnal sleep phase and increases during the wake phase repeatedly in a

\section{Biomed Central}


24-hour circadian rhythm. Sleep is most likely to occur when Tcore decreases, while it hardly occurs during the increasing phases [7]. This relationship between the sleepwake rhythm and the circadian rhythm of Tcore is important for maintaining sleep. At the normal sleep onset period in humans, Tcore decreases due to an underlying circadian rhythm, and sleep further induces this effect [8]. The driving force behind this Tcore decrease is the peripheral skin temperature (Tsk), which is rich in arteriovenous anastomoses and plays a central role in thermoregulation by adjusting blood flow to the skin [9]. Increased peripheral Tsk is largely due to reduced activation of noradrenergic vasoconstrictor tone, allowing greater inflow of heated blood from the core, thus facilitating heat loss to the environment through the skin surface [10]. The selective vasodilation of distal skin regions promotes the rapid onset of sleep [9] and is strongly associated with melatonin secretion [11]. Indeed, foot Tsk warming has been shown to reduce sleep onset latency [12], indicating that normal sleep onset is accomplished by increased peripheral heat loss and/or Tcore decrease [13]. The Tcore decrease in the sleep onset period is also strongly associated with cardiac autonomic activity. It has been suggested that changes in the cardiac autonomic nervous system precede sleep onset, which is strongly associated with changes in body temperature [14].

After sleep onset, Tcore gradually decreases further [8], while distal and proximal Tsk remain high [15]. The importance of maintaining Tsk at physiological range for sleep maintenance has been suggested [6]. In the elderly, only chest Tsk shows a significant decrease during sleep compared with the young $[16,17]$, and slow wave sleep (SWS) decreases and wakefulness increases compared with the young, with increased insomnia [18]. The sleep parameters measured by actigraphy correlate only with chest Tsk, and decreased chest Tsk is associated with a decrease in the sleep efficiency index [19]. Interestingly, even a slight increase in proximal Tsk increases the amount of SWS and decreases the early-morning awakening in the elderly [20]. Sleep-related areas in the brain are associated with an increased Tsk within the physiological range during sleep [21]. Moreover, Tsk might act as an input signal to sleep-regulating systems [6]. These results indicate that slightly increasing the proximal Tsk may help alleviate sleep problems, especially in the elderly. The temperature and humidity of the microclimate between humans and bed covers (bed climate) also play crucial roles in creating a warm bed climate temperature to support increased Tsk and sleep [6]. The bed climate temperature and relative humidity are generally maintained around $32^{\circ} \mathrm{C}$ to $34^{\circ} \mathrm{C}, 40 \%$ to $60 \%$ relative humidity when normal sleep is obtained $[22,23]$, which is in agreement with the comfort bed climate range suggested by Yanase [24].
Another important aspect to consider is that the thermoregulatory response during sleep differs depending on sleep stages. In animal studies, the thermoregulatory system is abolished during rapid eye movement sleep (REM) [25], due to a loss of thermosensitivity in the majority of the hypothalamic preoptic neurons [26]. In humans, thermosensitivity during REM is not completely depressed; however, sensitivity to hot or cold stimulation is reduced in REM compared to non-REM and wakefulness $[27,28]$. In addition, the sweat rate increases during SWS compared to other sleep stages [29], while delayed onset of sweating [30] and a decreased sweat rate [31] decrease evaporative heat dissipation and reduce heat tolerance during REM. Interestingly, the decreased sweat rate during REM is observed prior to REM stage onset [32]. Considering that skin sympathetic nerve activity (SSNA) contains sudomotor activity synchronous with vasodilator activity [33], this result indicates that SSNA might precede the sleep stage shift corresponding with heart rate variability (HRV) preceding the sleep stage shift at the sleep onset period [14]. Changes in the sensitivity of the sweat response depending on sleep stages are considered to be a central drive effect, since no peripheral change in sweat gland levels has been observed [34]. In cold exposure, shivering during sleep is confined to stages 1 and 2 and is not observed in SWS and REM [35], while the Tsk of the extremities is decreased during REM compared to that at control conditions [36]. These results indicate that REM and thermoregulation are mutually exclusive and partly explain the decrease in REM observed during heat or cold exposure, bearing in mind that REM is more sensitive to Ta than other sleep stages.

Although physiological thermoregulation related to sleep has been well defined, behavioral thermoregulation during sleep remains unclear. Limited behavioral thermoregulation reduces the thermoregulatory response during sleep compared to wakefulness. However, Ta increases or decreases during sleep significantly decrease or increase, respectively, the areas of the body covered by bed covers, with the neck, shoulder and upper extremities showing higher sensitivity than lower extremities and the trunk [37]. In heat exposure, lateral body position increases compared to the supine position, possibly because this position may decrease the contact area between the body and the mattress [38]. These results suggest that behavioral thermoregulation is active during sleep and that bed cover behaviors and body position may have an important role. Considering that poor sleepers spend more time on their backs with their heads straight, sleep positions may be related to sleep quality [39]. It is interesting to consider these behaviors in various thermal environments during sleep, since it may be an important sleep variable that would aid in our further 
understanding of thermoregulation during sleep in humans.

\section{Effects of heat exposure}

Increases in wakefulness are greater in cold Ta than in heat, suggesting that the impact of cold exposure is greater than that of heat exposure. Ta higher or lower than the thermal neutral temperature $\left(29^{\circ} \mathrm{C}\right)$ have been shown to increase wakefulness and decrease REM and SWS in semi-nude subjects [40]. However, these results are based on semi-nude subjects and exclude the effects of bed covers and clothing. In real-life situations where bed covers and clothing are used, sleep is actually disturbed during heat exposure rather than cold exposure in the young [41], as well as in the elderly [19]. The increased wakefulness and decreased SWS and REM are stereotypical effects that are observed in heat exposure [42]. These effects on sleep stages are concentrated in the initial segment rather than the later segment of sleep. One possible explanation for this is that sleep disruption in the initial sleep segment leads to an increased demand, which may overcome the thermal stress in the later segment of sleep [42,43]. Heat-related sleep disruptions do not adapt even after 5 days of continuous daytime and nocturnal heat exposure [44]. Furthermore, the effect on SWS does not change after partial sleep deprivation where sleep pressure is increased [45]. These results suggest a strong effect of heat load on sleep stages, which is related to thermoregulation during sleep. Thus, wakefulness is the only stage that can cope with an increased thermal load [26] and that wakefulness replaces SWS and REM to maintain homeothermy.

Heat load suppresses the decrease in Tcore and increases Tsk and whole body sweat loss during sleep $[43,46]$. Although Tsk increases at the onset of sleep, high Ta suppresses heat loss to the environment through the skin surface, thereby suppressing the decrease in Tcore. This suppressed decrease in Tcore may disturb sleep at the initial segment of sleep. The increased Tsk is largely due to increased skin blood flow, which is regulated primarily through two pathways in the sympathetic nervous system: the noradrenergic vasoconstrictor system and the active vasodilator system. Increased Tsk during sleep in heat exposure may be largely due to an increased active vasodilator system. In subjects that are awake, an active vasodilator system is responsible for most of the vasodilatory responses to heat stress [47]. The SSNA contains vasodilator activity synchronous with sudomotor activity [33] and may lead to an increase in whole body sweat loss. Taken together, these results suggest the possibility that increased vasodilator activity may be related to increased Tsk and wakefulness due to heat exposure during sleep. Because sleep distribution is controlled by both peripheral and central driving effects
$[13,48]$, it is possible that increased active vasodilation and Tcore both increase wakefulness during heat exposure. These results support the notion that, although sleep states affect thermoregulation, thermoregulation equally affects the mechanism governing sleep [1].

One of the most important factors that increase heat stress during sleep is the humidity. Humid heat exposure further increases wakefulness, decreases REM and SWS, and excessively suppresses the decrease in Tcore, whereas Tsk and whole body sweat loss are not affected [43]. Humid heat exposure most probably increases heat stress because of the difference in the sweat response caused by the humidity. Decreased ambient humidity allows sweat to evaporate, thereby dissipating the heat, whereas increased humidity does not allow the sweat to evaporate, causing the skin to remain wet. The dripping sweat and increased skin wetness decrease the sweat response due to hidromeiosis preventing dehydration. These results indicate the importance of taking humidity into account, especially in Japan and many other Asian countries that experience humid heat in the summer.

Interestingly, although the exposure time is the same, the effect of humid heat is greater in the initial segment than in the later segment of sleep. Humid heat exposure during the later segment of sleep increases wakefulness in that segment $[49,50]$. In contrast, humid heat exposure in the initial segment decreases SWS in that segment and increases wakefulness in both segments [50]. Furthermore, decreased SWS in the initial segment of sleep tends to increase it in the later segment of sleep. Thus, humid heat exposure in the initial segment of sleep appears to change the polarity of SWS [50]. These effects on sleep stages can be explained by thermoregulation and microclimate temperature (temperature and humidity of the microclimate between skin and the clothing) (Figure 1). Humid heat exposure in the later segment causes a decrease in Tcore in the initial segment, followed by an increase in Tcore, Tsk and microclimate temperature in the later segment. Humid heat exposure in the initial segment suppresses the decrease in Tcore, whereas Tsk and the microclimate temperature increase lead to a decrease in SWS and an increase in wakefulness. Furthermore, the sharp decrease in Tcore, Tsk and the microclimate temperature in the later segment of sleep may also increase wakefulness. The chilling effects of a decrease in $\mathrm{Ta}$ and humidity and a reduction in clothing insulation due to wetness [51] caused by sweating at initial humid heat exposure may also be a possibility for the increase in wakefulness. Another possible contributing factor for decreased Tsk and Tcore in the later segment of sleep could be that a decrease in Ta coincides with a time when REM sleep generally increases, since Tsk decreases are greater in REM compared to other sleep stages [36]. In Japan, a majority 


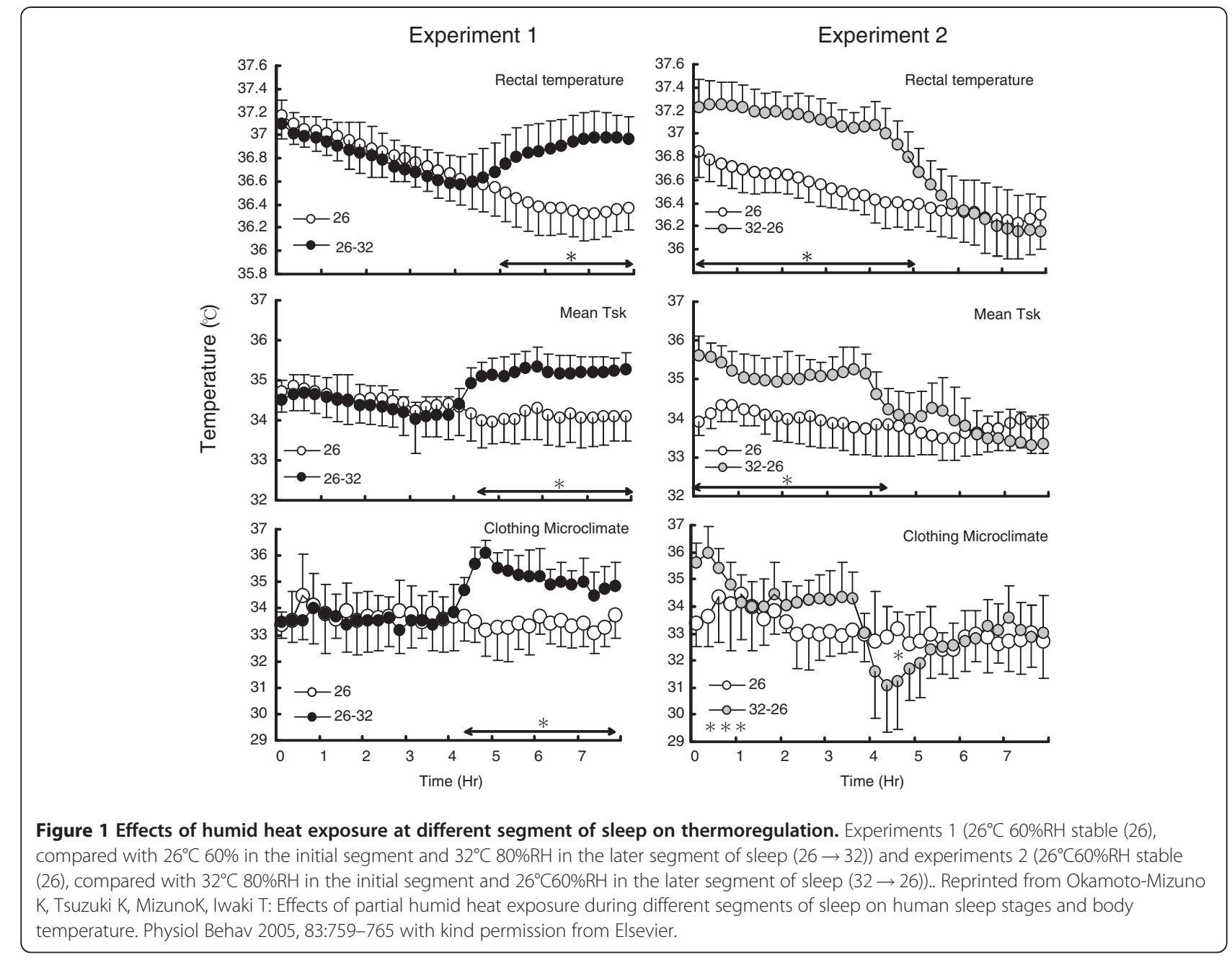

use air conditioning only for a few hours after going to sleep [52] due to the notion that being exposed to air conditioning is unhealthy [53], and 90\% of Japanese people are interested in saving energy [54]. These results indicate that if air conditioning use is limited, then it should be used during the initial segment of sleep. Furthermore, when air conditioning is used in the later segment of sleep, drying off the sweat and changing clothing are essential to avoid chilling effects.

In the elderly, even mild heat exposure increases wakefulness and decreases REM [55]. Since the elderly exhibit a decreased amount of SWS even under normal conditions, SWS is not thought to be affected by heat exposure. Sleep consolidation decreases in older men [56], which in turn increases susceptibility to external arousal stimuli during sleep [57]. These changes in sleep may decrease the thermal awakening threshold. Another reason for this could be reduced heat tolerance in older men, since most heat exposure studies have found a reduced ability to regulate Tcore in subjects who are awake (for example, see [58]). Mild heat exposure further suppresses the decline in Tcore, increases Tsk, and increases whole body sweat loss by two-fold in the elderly [55]. The increase in nocturnal Tcore [59] and the attenuation of the nocturnal drop in Tcore [60] may underlie age-related declines in sleep maintenance and sleep quality. Furthermore, the period of overnight fasting results in mild dehydration in older men even under normal conditions [61], suggesting that increased whole body sweat loss during sleep in mild heat exposure may be related to dehydration in the elderly. Although further study to directly compare older and younger men is needed, it appears quite likely that sleep in older men is more affected by heat exposure than in younger men. These results indicate that the Ta during sleep warrants particularly careful consideration in older men, especially since decreased sleep duration in the older men is related to reduced quality of life and mortality $[3,4]$. Ta for the elderly should also take into account clothing conditions; elderly Japanese people wear more than two layers of underwear under nightwear even in summer [62]. 


\section{Effects of cold exposure}

The difference between cold exposure and heat exposure is that cold exposure mainly affects the later segment of sleep, where REM is dominant [63]. In semi-nude subjects, cold exposure mainly affects REM due to suppression of the thermoregulatory response [35,40]. SWS is not affected because it predominates in the initial segment of sleep [64]. In thermoregulation during sleep, Tcore decreases through the night [36] as the Ta decreases [65]. However, in real-life situations people generally use clothing and bed covers during sleep in cold exposure. In studies using clothing and/or bedding, no significant difference was observed in sleep in a Ta range of $13^{\circ} \mathrm{C}$ to $23^{\circ} \mathrm{C}$ [66] and $3^{\circ} \mathrm{C}$ to $17^{\circ} \mathrm{C}$ [67]. Also, no significant difference in sleep quality measured by actigraphy was observed between $9^{\circ} \mathrm{C}$ and $20^{\circ} \mathrm{C}$ in the elderly [19]. These results indicate that, in real-life situations, cold exposure does not affect sleep. This is related to the fact that despite large changes in Ta the bed climate temperature remained relatively constant [66]. The use of bed covers allows for the development of an isolated high bed climate temperature, which is critical for maintaining sleep [6] as well as determining sleep quality [68].

However, our study indicates that cold exposure significantly changes cardiac autonomic activity during sleep, without affecting the sleep stages (Figure 2) [67]. With regard to cardiac autonomic activity based on the HRV index, the ratio of the low frequency (LF) to high frequency (HF) band (LF/HF) significantly decreases during stage 2 and SWS, while the percentage of the LF component $(\mathrm{LF} /(\mathrm{LF}+\mathrm{HF}))$ significantly decreases during SWS as the Ta decreases from $17^{\circ} \mathrm{C}$ to $3^{\circ} \mathrm{C}$. In contrast, no significant effect is observed during REM or wakefulness. These results may indicate that cardiac parasympathetic activity predominates under cold exposure during stage 2 and SWS, although the results of the LF/HF and $\mathrm{LF} /(\mathrm{LF}+\mathrm{HF})$ should be interpreted with caution. The dominant parasympathetic activity during stage 2 and SWS may be related to at least three factors: cold stimulation of the head since sufficient thermal insulation of the body is obtained from bedding and clothing; cold air inhalation; and whole body cooling. First, cold stimulation of the face, a unique reflex referred to as cold face test, increases the cardiac parasympathetic activity and the peripheral skin SSNA simultaneously and integrates the trigeminal brain stem reflex arc in wakeful subjects [69]. Additionally, this reflex activates reflex centers located in the medullary region and induces bradycardia [70-72]. Furthermore, the concomitant increase in the SSNA leads to vasoconstriction, an increase in blood pressure [70-72] and a significant increase in the HF component $[69,73]$. Second, inhalation of cold air may increase muscle sympathetic nervous activity and blood pressure in wakeful subjects [74]. Third, whole body

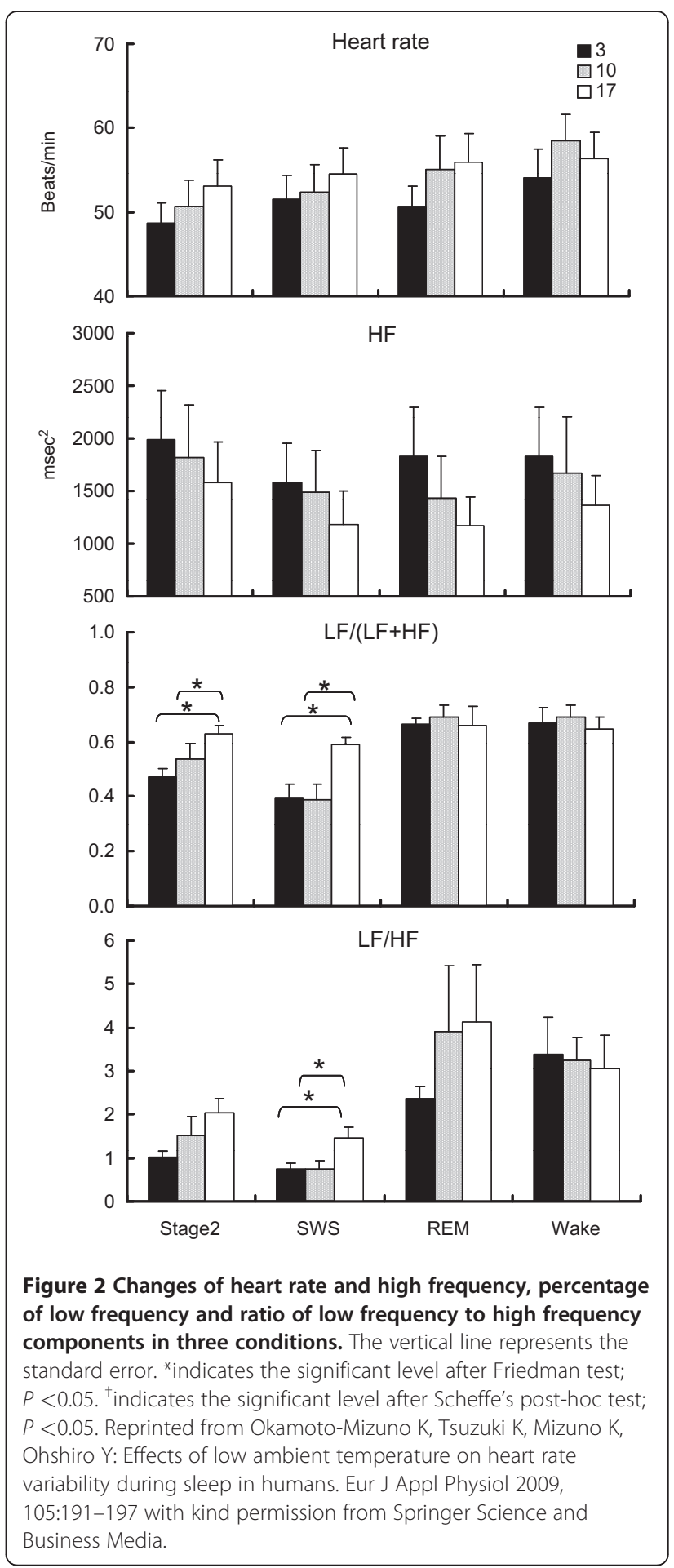

cooling may also be related to increases in systolic and diastolic blood pressure and decreases in heart rate in wakeful subjects [75]. Considering that approximately $70 \%$ of sleep time comprises stage 2 and SWS, the cardiac parasympathetic activity may be dominant during sleep in cold exposure. 
These findings could partly explain the adverse cardiac events that peak during the colder periods in the winter season [76,77]. Mortality due to ischemic heart disease is not related to low outside temperature, but to low living-room temperatures and limited bedroom heating in the winter [78]. Cold climates increase blood pressure [75] as well as the levels of hematological factors that favor arterial thrombosis [79] and fibrinogen synthesis [80]. It has been suggested that the onset of major cardiovascular events is triggered by sleep stage-dependent fluctuations in the autonomic nervous system [81]. Significantly dominant cardiac parasympathetic activity during stage 2 and SWS, which is not observed during REM and wakefulness in cold exposure, suggests marked variations in HRV during the transition from stage 2 to REM and wakefulness. The marked rise in HRV during transition from non-REM to awakening and REM favors adverse cardiac events [82]. Further study considering the relationship between the increased incidence of adverse cardiac events and marked variations in HRV during transition from non-REM to wakefulness and REM may provide insights into understanding the increase in cardiac events peaking in winter. Besides HRV, the lack of nocturnal decline in blood pressure is also related to an increase in end-organ damage and cardiovascular events $[83,84]$. Considering that the cold face test, inhaling cold air and whole body cooling accompany increased blood pressure, it would be interesting to investigate whether cold exposure suppresses blood pressure decline during sleep. It is extremely important to note that $\mathrm{Ta}$ in winter should be maintained at a level higher than $10^{\circ} \mathrm{C}$. However, the most difficult aspect of cold exposure is that sleep is not disturbed. The impact on the cardiovascular response may be occurring without subjective sensation, suggesting that cold exposure may have more impact than heat exposure. In Japan, bedroom Ta falls to as low as $3^{\circ} \mathrm{C}$ in the suburbs [53]. Excessive layers of underwear under night wear and bed covers are common observations in elderly Japanese people [85] and this behavior supports sleep in cold exposure. Indeed, about $50 \%$ of elderly Japanese people use two to six layers of underwear and three to five layers of bed covers [85]. The most important aspect of this is whether HRV during sleep in elderly people habitually sleeping in cold Ta is affected by cold exposure or is somewhat adapted to it. Further study on measuring HRV in the elderly habitually sleeping in cold Ta at home may thus be required.

\section{Ambient temperature and circadian rhythm}

The environmental light-dark cycle is the principal environmental synchronizer of the circadian pacemaker in humans as well as other species [7]; however, the effects of social cues and other nonphotic entrainment, including Ta, on human circadian system are less understood.
Twenty-four hours of warm Ta increases activity, suggesting that Ta has a masking effect on circadian activity rhythms in animal studies [86]. In high and cool Ta cycles, Ta acts as a weak synchronizer in laboratory rats [87] and mice [88]. These results suggest that, at the least, Ta may have masking effects on circadian activity rhythms in homeothermic animals. In humans, many studies indicate that different Ta cycles during sleep within the thermoneutral Ta range can affect Tcore. Decreased Ta occurring a few hours before and after sleep onset and increased Ta occurring around wake-up time increases the decline in Tcore and advances the Tcore nadir compared to constant Ta $[89,90]$ and/or the opposite Ta cycle $[91,92]$. The effects of these cyclic Ta changes do not significantly affect sleep stages [89], or increase SWS compared to constant Ta [90]. These results indicate that cyclic Ta changes do not induce any adverse effects on sleep stages at least within the thermoneutral temperature range. Interestingly, studies by Dewasmes et al. [89,93] showed that cyclic Ta, as opposed to constant Ta, advanced the minimum Tcore by 143 minutes and the propensity for REM. The REM cycle length changes depending on Ta with a delayed REM cycle in low Ta compared with high Ta [94], and the REM propensity has a close relationship with body temperature rhythm [95]. These results indicate that Ta itself as well as cyclic Ta change may advance circadian Tcore and/or REM and that the thermoregulatory system may have effects on phase advancing mechanisms.

One possible explanation for these effects of cyclic Ta change on Tcore may be the reduced thermoregulation during sleep compared to wakefulness [28]. No significant difference between sleep stages at Ta of $13^{\circ} \mathrm{C}$ to $23^{\circ} \mathrm{C}$ is observed, although Tcore clearly decreases as the $\mathrm{Ta}$ decreases [66]. Not only Ta but also bed mattress properties with decreased thermal insulation result in significantly decreased Tcore without affecting sleep stages [96]. Although sleep stages were not measured, effects of different types of quilts [97] and clothing [98] during sleep decreased Tcore under decreased thermal insulation conditions. The effects of heat exposure during sleep are also greater compared to the waking state with an increase of a few degrees in Ta above the thermal neutral zone affecting Tcore during sleep [31]. These results indicate that Tcore during sleep may be sensitive to Ta change as well as clothing and bedding thermal insulation. It is important to note that these effects on Tcore do not always affect sleep stages in parallel. During cyclic Ta changes, Tcore may be affected by peripheral skin blood flow that regulates the circadian rhythm of Tcore [99]. Increased and decreased dry heat loss from changes in the peripheral Tsk has been suggested as one possible effect of cyclic Ta change on Tcore during sleep [100]. However, further precise study 
on peripheral Tsk under cyclic Ta changes together with sleep stages, especially with REM propensity and cycle, melatonin secretion and bed climate, is needed.

Interestingly, it has been suggested that time memory for heat exposure exists in the human thermoregulatory system, and that autonomic thermoregulation in Tcore changes during the previous heat exposure period without actual temperature stimuli [101]. It would be interesting to know whether continuous phase shifting effect of cyclic Ta changes during sleep may keep time memory and whether this phase shifting effect continues even under constant Ta. Furthermore, effects of cyclic Ta change on Tcore are limited during sleep, and further study is warranted to determine its effects on the wakeful state. Results from blind individuals indicate that, although nonphotic stimuli can exert a small, but significant resetting response, these effects are weaker than light stimuli in affecting the human circadian pacemaker [102]. Indeed, cyclic Ta change combined with a gradual light-dark intensity cycle indicates a stronger effect of light compared with Ta [103]. In real-life situations, Ta and light change in a 24-hour cycle may involve the circadian system in humans. It has been suggested that in, addition to light, the daily rise and fall in environmental temperature could be an essential input to the circadian clock [104]. Since thermoregulatory mechanisms are strongly related with the circadian timing system, the Ta change may be an essential entrainment input additional to light environment, or at least exert masking effects on the circadian system.

\section{Conclusions}

Heat exposure affects SWS and REM, whereas cold exposure does not affect sleep stages. Considering that a Ta of $32^{\circ} \mathrm{C}$ with $80 \%$ relative humidity affects only SWS without affecting REM [46], heat affects SWS first, whereas REM may be well-preserved in real-life situations. Sleep disturbance during heat exposure may lead to behavioral thermoregulation in humans, for example, using an air conditioner to decrease Ta. However, during cold exposure, the cardiac autonomic response may be affected without affecting sleep stages and subjective sensations, and so not trigger behavioral thermoregulation to control Ta. This indicates that the impact of cold exposure may be greater than that of heat; thus, further studies are warranted to consider the effect of cold exposure on sleep and other physiological parameters.

\section{Competing interests}

The authors declare that they have no competing interests.

\section{Authors' contributions}

KO-M has made substantial contributions to the manuscript. KM has been involved in drafting the manuscript and revising it critically for important intellectual content. Both authors read and approved the final manuscript.

\section{Author details}

${ }^{1}$ Kansei Fukushi Research Center, Tohoku Fukushi University, 1-149-6 Kunimigaoka Aoba Sendai, Miyagi, 981-0935, Japan. ²Department of Early Childhood and Primary Education, Tohoku Fukushi University, 6-149-1 Kunimi, Aoba-ku, Sendai, Miyagi, 989-3201, Japan

Received: 22 March 2012 Accepted: 31 May 2012 Published: 31 May 2012

\section{References}

1. Gilbert SS, van den Heuvel CJ, Ferguson SA, Dawson D: Thermoregulation as a sleep signalling system. Sleep Med Rev 2004, 8:81-93.

2. Hasler G, Buysse DJ, Klaghofer R, Gamma A, Ajdacic V, Eich D, Rossler W, Angst J: The association between short sleep duration and obesity in young adults: a 13-year prospective study. Sleep 2004, 27:661-666.

3. Dew MA, Hoch CC, Buysse DJ, Monk TH, Begley AE, Houck PR, Hall M, Kupfer DJ, Reynolds CF 3rd: Healthy older adults' sleep predicts all-cause mortality at 4 to 19 years of follow-up. Psychosom Med 2003, 65:63-73.

4. Manabe K, Matsui T, Yamaya M, Sato-Nakagawa T, Okamura N, Arai H, Sasaki H: Sleep patterns and mortality among elderly patients in a geriatric hospital. Gerontology 2000, 46:318-322.

5. Parsons K: Human Thermal Environments. Oxford: Taylor \& Francis; 1993.

6. Van Someren EJ: Mechanisms and functions of coupling between sleep and temperature rhythms. Prog Brain Res 2006, 153:309-324

7. Czeisler C, Buxton O, Khalsa S: The human circadian timing system and sleep-wake regulation. In Principles and Practice of Sleep Medicine. Edited by Kryger M, Roth T, Dement W. Philadelphia: Elsevier; 2005.

8. Barrett J, Lack L, Morris M: The sleep-evoked decrease of body temperature. Sleep 1993, 16:93-99.

9. Krauchi K, Cajochen C, Werth E, Wirz-Justice A: Functional link between distal vasodilation and sleep-onset latency? Am J Physiol Regul Integr Comp Physiol 2000, 278:R741-R748.

10. Lack $L$, Gradisar M: Acute finger temperature changes preceding sleep onsets over a 45-h period. J Sleep Res 2002, 11:275-282

11. Krauchi K, Cajochen C, Wirz-Justice A: Circadian and homeostatic regulation of core body temperature and alertness in humans: what is the role of melatonin? In Circadian Clocks and Entrainment. Edited by Honma K, Honma S. Hokkaido: Hokkaido University Press; 1998.

12. Raymann RJ, Swaab DF, van Someren EJ: Skin temperature and sleep-onset latency: changes with age and insomnia. Physiol Behav 2007, 90:257-266.

13. Van Someren EJ: More than a marker: interaction between the circadian regulation of temperature and sleep, age-related changes, and treatment possibilities. Chronobiol Int 2000, 17:313-354.

14. Okamoto-Mizuno K, Yamashiro Y, Tanaka H, Komada Y, Mizuno K, Tamaki M, Kitado $M$, Inoue $Y$, Shirakawa S: Heart rate variability and body temperature during the sleep onset period. Sleep Biol Rhythms 2008, 6:42-49.

15. Krauchi K, Knoblauch V, Wirz-Justice A, Cajochen C: Challenging the sleep homeostat does not influence the thermoregulatory system in men: evidence from a nap vs. sleep-deprivation study. Am J Physiol Regul Integr Comp Physiol 2006, 290:R1052-R1061.

16. Okamoto $K$, Nagai $Y$, lizuka S: Age effects on physiological responses and bed climate during sleep after heating the lower extremities. $J$ Home Economics Japan 1998, 49:1307-1312.

17. Okamoto K, Nagai Y, lizuka S: Effects of age on physiological response and bed climate during sleep followed by using the electric blanket. $J$ Home Economics Japan 1999, 50:259-265.

18. Monk TH, Reynolds CF 3rd, Buysse DJ, Hoch CC, Jarrett DB, Jennings JR, Kupfer DJ: Circadian characteristics of healthy 80 -year-olds and their relationship to objectively recorded sleep. J Gerontol 1991, 46:M171-M175.

19. Okamoto-Mizuno K, Tsuzuki K: Effects of season on sleep and skin temperature in the elderly. Int J Biometeorol 2010, 54:401-409.

20. Raymann RJ, Swaab DF, van Someren EJ: Skin deep: enhanced sleep depth by cutaneous temperature manipulation. Brain 2008, 131:500-513.

21. Van Someren E: Sleep propensity is modulated by circadian and behaviorinduced changes in cutaneous temperature. J Thermal Biol 2004, 29:437-444.

22. Okamoto K, lizuka S, Okudaira N: The effects of air mattress upon sleep and bed climate. Appl Human Sci 1997, 16:97-102.

23. Okamoto K, Mizuno K, Okudaira N: The effects of a newly designed air mattress upon sleep and bed climate. Appl Human Sci 1997, 16:161-166. 
24. Yanase T: A study on the physiological and psychological comfort of residential conditions. J Home Economics Jap 1998, 49:975-984

25. Glotzbach SF, Heller HC: Central nervous regulation of body temperature during sleep. Science 1976, 194:537-539.

26. Parmeggiani PL: Interaction between sleep and thermoregulation: an aspect of the control of behavioral states. Sleep 1987, 10:426-435.

27. Candas V, Libert JP, Muzet A: Heating and cooling stimulations during SWS and REM sleep in man. J Therm Biol 1982, 7:155-158.

28. Jennings JR, Reynolds CF, Bryant DS, Berman SR, Buysse DJ, Dahl RE, Hoch CC, Monk TH: Peripheral thermal responsivity to facial cooling during sleep. Psychophysiology 1993, 30:374-382.

29. Ogawa T, Satoh T, Takagi K: Sweating during night sleep. Jpn J Physiol 1967, 17:135-148.

30. Libert JP, Candas V, Muzet A, Ehrhart J: Thermoregulatory adjustments to thermal transients during slow wave sleep and REM sleep in man. J Physiol Paris 1982, 78:251-257.

31. Sagot JC, Amoros C, Candas V, Libert JP: Sweating responses and body temperatures during nocturnal sleep in humans. Am J Physiol 1987, 252: R462-R470.

32. Dewasmes G, Bothorel B, Candas V, Libert JP: A short-term poikilothermic period occurs just after paradoxical sleep onset in humans: characterization changes in sweating effector activity. J Sleep Res 1997, 6:252-258.

33. Sugenoya J, Iwase S, Mano T, Sugiyama Y, Ogawa T, Nishiyama T, Nishimura N, Kimura T: Vasodilator component in sympathetic nerve activity destined for the skin of the dorsal foot of mildly heated humans. J Physiol 1998, 507(Pt 2):603-610.

34. Amoros C, Sagot JC, Libert JP, Candas V: Sweat gland response to local heating during sleep in man. J Physiol Paris 1986, 81:209-215.

35. Haskell EH, Palca JW, Walker JM, Berger RJ, Heller HC: Metabolism and thermoregulation during stages of sleep in humans exposed to heat and cold. J Appl Physiol 1981, 51:948-954.

36. Palca JW, Walker JM, Berger RJ: Thermoregulation, metabolism, and stages of sleep in cold-exposed men. J Appl Physiol 1986, 61:940-947.

37. Okamoto-Mizuno $K$, Nagai $Y$, lizuka S: The effects of ambient temperature change on the covered area of the body during sleep. J Home Economics Jap 2003, 54:1025-1030.

38. Miyazawa M: On the correlation between bed climate influenced by combinations of mattresses and sleep. Res I Living Science 1976, 23:86-91.

39. De Koninck J, Gagnon P, Lallier S: Sleep positions in the young adult and their relationship with the subjective quality of sleep. Sleep 1983, 6:52-59.

40. Haskell EH, Palca JW, Walker JM, Berger RJ, Heller HC: The effects of high and low ambient temperatures on human sleep stages.

Electroencephalogr Clin Neurophysiol 1981, 51:494-501.

41. Okamoto-Mizuno K, Mizuno K: Sleep and environment. Treatment Strageties - Respiratory 2011, 2:87-89.

42. Karacan I, Thornby Jl, Anch AM, Williams RL, Perkins HM: Effects of high ambient temperature on sleep in young men. Aviat Space Environ Med 1978, 49:855-860.

43. Okamoto-Mizuno K, Mizuno K, Michie S, Maeda A, lizuka S: Effects of humid heat exposure on human sleep stages and body temperature. Sleep 1999, 22:767-773.

44. Libert JP, Di Nisi J, Fukuda H, Muzet A, Ehrhart J, Amoros C: Effect of continuous heat exposure on sleep stages in humans. Sleep 1988, 11:195-209.

45. Bach V, Maingourd Y, Libert JP, Oudart H, Muzet A, Lenzi P, Johnson LC: Effect of continuous heat exposure on sleep during partial sleep deprivation. Sleep 1994, 17:1-10.

46. Tsuzuki K, Okamoto-Mizuno K, Mizuno K: Effects of humid heat exposure on sleep, thermoregulation, melatonin, and microclimate. J Therm Biol 2004, 29:31-36.

47. Pierzga JM, Frymoyer A, Kenney WL: Delayed distribution of active vasodilation and altered vascular conductance in aged skin. $J$ Appl Physiol 2003, 94:1045-1053.

48. Sakaguchi S, Glotzbach SF, Heller HC: Influence of hypothalamic and ambient temperatures on sleep in kangaroo rats. Am J Physiol 1979, 237: R80-R88.

49. Okamoto-Mizuno K, Tsuzuki K, Mizuno K: Effects of humid heat exposure in later sleep segments on sleep stages and body temperature in humans. Int I Biometeorol 2005, 49:232-237.

50. Okamoto-Mizuno K, Tsuzuki K, Mizuno K, Iwaki T: Effects of partial humid heat exposure during different segments of sleep on human sleep stages and body temperature. Physiol Behav 2005, 83:759-765.
51. Bakkevig MK, Nielsen R: Impact of wet underwear on thermoregulatory responses and thermal comfort in the cold. Ergonomics 1994, 37:1375-1389.

52. Miyazawa M: Air conditioning. In Sleep Environment. Tokyo: Asakura Shoten; 1999:157-163 [Torii S (Series Editor)]

53. Tsuzuki K, Yokoyama K, Yokoi T, Ogi H, Taya H, Yoshioka M, Nakamura K: Thermal environments and indoor air quality in the elderly houses in a rural area. Jpn J Biometeor 2001, 38:23-32.

54. Sassa N, Isoda N: Study on the consciousness of energy-saving and life style - a case for the detached house of the newly-developed residential area in the suburbs of Nara city. J Home Economics Jap 2003, 54:935-943.

55. Okamoto-Mizuno K, Tsuzuki K, Mizuno K: Effects of mild heat exposure on sleep stages and body temperature in older men. Int J Biometeorol 2004 49:32-36.

56. Dijk DJ, Duffy JF, Riel E, Shanahan TL, Czeisler CA: Ageing and the circadian and homeostatic regulation of human sleep during forced desynchrony of rest, melatonin and temperature rhythms. J Physiol 1999, 516(Pt 2):611-627.

57. Dijk DJ, Duffy JF, Czeisler CA: Contribution of circadian physiology and sleep homeostasis to age-related changes in human sleep. Chronobiol Int 2000, 17:285-311.

58. Inoue $Y$, Kuwahara T, Araki T: Maturation- and aging-related changes in heat loss effector function. J Physiol Anthropol Appl Human Sci 2004, 23:289-294

59. Lushington K, Dawson D, Lack L: Core body temperature is elevated during constant wakefulness in elderly poor sleepers. Sleep 2000, 23:504-510.

60. Dawson D, Rogers NL, van den Heuvel CJ, Kennaway DJ, Lushington K: Effect of sustained nocturnal transbuccal melatonin administration on sleep and temperature in elderly insomniacs. J Biol Rhythms 1998, 13:532-538.

61. Kenney WL, Chiu P: Influence of age on thirst and fluid intake. Med Sci Sports Exerc 2001, 33:1524-1532.

62. Okamoto K, Kudoh Y, Yokoya T, Okudaira N: A survey of bedroom and bed climate of the elderly in a nursing home. Appl Human Sci 1998, 17:115-120

63. Okamoto-Mizuno K, Tsuzuki K, Ohshiro Y, Mizuno K: Effects of an electric blanket on sleep stages and body temperature in young men. Ergonomics 2005, 48:749-757.

64. Buguet A, Cespuglio R, Radomski MW: Sleep and stress in man: an approach through exercise and exposure to extreme environments. Can J Physiol Pharmacol 1998, 76:553-561.

65. Kreider MB, lampietro PF: Oxygen consumption and body temperature during sleep in cold environments. J Appl Physiol 1959, 14:765-767.

66. Muzet A, Libert JP, Candas V: Ambient temperature and human sleep. Experientia 1984, 40:425-429.

67. Okamoto-Mizuno K, Tsuzuki K, Mizuno K, Ohshiro Y: Effects of low ambient temperature on heart rate variability during sleep in humans. Eur J Appl Physiol 2009, 105:191-197.

68. Candas V, Libert J, Vogt J, Ehrhart J, Muzet A: Body temperature during sleep under different thermal conditions. In Indoor Climate, Effect on Human Comfort, Performance and health:: Danish Building Research Institute; 1979:763-776

69. Hilz MJ, Stemper B, Sauer P, Haertl U, Singer W, Axelrod FB: Cold face test demonstrates parasympathetic cardiac dysfunction in familial dysautonomia. Am J Physiol 1999, 276:R1833-R1839.

70. Brown CM, Sanya EO, Hilz MJ: Effect of cold face stimulation on cerebral blood flow in humans. Brain Res Bull 2003, 61:81-86.

71. Gavhed D, Makinen T, Holmer I, Rintamaki H: Face temperature and cardiorespiratory responses to wind in thermoneutral and cool subjects exposed to -10 degrees C. Eur J Appl Physiol 2000, 83:449-456.

72. LeBlanc J, Mercier I: Cold wind stimulation reflex. J Appl Physiol 1992, 73:1704-1707

73. Heart rate variability: standards of measurement, physiological interpretation and clinical use. Task Force of the European Society of Cardiology and the North American Society of Pacing and Electrophysiology. Circulation 1996, 93:1043-1065.

74. Heindl S, Struck J, Wellhoner P, Sayk F, Dodt C: Effect of facial cooling and cold air inhalation on sympathetic nerve activity in men. Respir Physiol Neurobiol 2004, 142:69-80.

75. Korhonen I: Blood pressure and heart rate responses in men exposed to arm and leg cold pressor tests and whole-body cold exposure. Int $J$ Circumpolar Health 2006, 65:178-184. 
76. Kloner RA: Natural and unnatural triggers of myocardial infarction. Prog Cardiovasc Dis 2006, 48:285-300.

77. Mercer JB: Cold-an underrated risk factor for health. Environ Res 2003, 92:8-13.

78. Cold exposure and winter mortality from ischaemic heart disease, cerebrovascular disease, respiratory disease, and all causes in warm and cold regions of Europe. The Eurowinter Group. Lancet 1997, 349:1341-1346.

79. Keatinge WR, Coleshaw SR, Cotter F, Mattock M, Murphy M, Chelliah R: Increases in platelet and red cell counts, blood viscosity, and arterial pressure during mild surface cooling: factors in mortality from coronary and cerebral thrombosis in winter. Br Med J (Clin Res Ed) 1984, 289:1405-1408.

80. Rudnicka AR, Rumley A, Lowe GD, Strachan DP: Diurnal, seasonal, and bloodprocessing patterns in levels of circulating fibrinogen, fibrin D-dimer, C-reactive protein, tissue plasminogen activator, and von Willebrand factor in a 45-year-old population. Circulation 2007, 115:996-1003.

81. Lavery CE, Mittleman MA, Cohen MC, Muller JE, Verrier RL: Nonuniform nighttime distribution of acute cardiac events: a possible effect of sleep states. Circulation 1997, 96:3321-3327.

82. Viola AU, Simon C, Ehrhart J, Geny B, Piquard F, Muzet A, Brandenberger G: Sleep processes exert a predominant influence on the 24 -h profile of heart rate variability. J Biol Rhythms 2002, 17:539-547.

83. Ohkubo T, Hozawa A, Yamaguchi J, Kikuya M, Ohmori K, Michimata M, Matsubara M, Hashimoto J, Hoshi H, Araki T, Tsuji I, Satoh H, Hisamichi S, Imai Y: Prognostic significance of the nocturnal decline in blood pressure in individuals with and without high $24-\mathrm{h}$ blood pressure: the Ohasama study. J Hypertens 2002, 20:2183-2189.

84. Verdecchia P, Porcellati C, Schillaci G, Borgioni C, Ciucci A, Battistelli M, Guerrieri M, Gatteschi C, Zampi I, Santucci A, Reboldi G: Ambulatory blood pressure. An independent predictor of prognosis in essential hypertension. Hypertension 1994, 24:793-801.

85. Okamoto K, Kimura F, Tsuruhashi R, lizuka S: A Survey of sleep environment of elderly people. Proceedings of the Jissen Women's University 1993, 30:63-69.

86. Aschoff J, Tokura H: Circadian activity rhythms in squirrel monkeys: entrainment by temperature cycles. J Biol Rhythms 1986, 1:91-99.

87. Francis AJ, Coleman GJ: The effect of ambient temperature cycles upon circadian running and drinking activity in male and female laboratory rats. Physiol Behav 1988, 43:471-477.

88. Refinetti R: Entrainment of circadian rhythm by ambient temperature cycles in mice. J Biol Rhythms 2010, 25:247-256.

89. Dewasmes G, Nicolas A, Rodriguez D, Salame P, Eschenlauer R, Joly D, Muzet $A$ : Human core temperature minimum can be modified by ambient thermal transients. Neurosci Lett 1994, 173:151-154.

90. Togo F, Aizawa S, Arai J, Yoshikawa S, Ishiwata T, Shephard RJ, Aoyagi Y: Influence on human sleep patterns of lowering and delaying the minimum core body temperature by slow changes in the thermal environment. Sleep 2007, 30:797-802.

91. Teramoto Y, Oikura H, loki I, Suho S, Inoshiri R, Masuda M: The effect of room temperature on rectal temperature during night sleep. $J$ Therm Biol 1998, 23:15-21.

92. Wakamura $\mathrm{T}$, Tokura $\mathrm{H}$ : Circadian rhythm of rectal temperature in humans under different ambient temperature cycles. J Therm Biol 2002, 27:439-447.

93. Dewasmes G, Signoret P, Nicolas A, Ehrhart J, Muzet A: Advances of human core temperature minimum and maximal paradoxical sleep propensity by ambient thermal transients. Neurosci Lett 1996, 215:25-28.

94. Muzet A, Ehrhart J, Candas V, Libert J, Vogt J: REM sleep and ambient temperature in man. Int J Neuroscience 1983, 18:117-125.

95. Czeisler CA, Zimmerman JC, Ronda JM, Moore-Ede MC, Weitzman ED: Timing of REM sleep is coupled to the circadian rhythm of body temperature in man. Sleep 1980, 2:329-346.

96. Okamoto K, Nakabayashi K, Mizuno K, Okudaira N: Effects of truss mattress upon sleep and bed climate. Appl Human Sci 1998, 17:233-237.

97. Okada M, Midorikawa-Tsurutani T, Tokura H: The effects of two different kinds of quilt on human core temperature during night sleep. Ergonomics 1994, 37:851-857.

98. Park SJ, Tokura H: Effects of different types of clothing on circadian rhythms of core temperature and urinary catecholamines. Jpn J Physiol 1998, 48:149-156.

99. Krauchi K, Wirz-Justice A: Circadian rhythm of heat production, heart rate, and skin and core temperature under unmasking conditions in men. Am J Physiol 1994, 267:R819-R829.
100. Kondo M, Tokura H, Wakamura T, Hyun KJ, Tamotsu S, Morita T, Oishi T: Physiological significance of cyclic changes in room temperature around dusk and dawn for circadian rhythms of core and skin temperature, urinary 6-hydroxymelatonin sulfate, and waking sensation just after rising. J Physiol Anthropol 2007, 26:429-436.

101. Shido O, Sugimoto N, Tanabe M, Sakurada S: Core temperature and sweating onset in humans acclimated to heat given at a fixed daily time. Am J Physiol 1999, 276:R1095-R1101.

102. Klerman EB, Rimmer DW, Dijk DJ, Kronauer RE, Rizzo JF 3rd, Czeisler CA: Nonphotic entrainment of the human circadian pacemaker. Am J Physiol 1998, 274:R991-R996.

103. Kondo M, Tokura H, Wakamura T, Hyun KJ, Tamotsu S, Morita T, Oishi T: Combined influences of gradual changes in room temperature and light around dusk and dawn on circadian rhythms of core temperature, urinary 6-hydroxymelatonin sulfate and waking sensation just after rising. Coll Antropol 2007, 31:587-593.

104. Van Someren E: Thermosensitivity of the circadian timing system. Sleep and Biological Rhythms 2003, 1:55-64.

doi:10.1186/1880-6805-31-14

Cite this article as: Okamoto-Mizuno and Mizuno: Effects of thermal environment on sleep and circadian rhythm. Journal of Physiological Anthropology 2012 31:14

\section{Submit your next manuscript to BioMed Central and take full advantage of:}

- Convenient online submission

- Thorough peer review

- No space constraints or color figure charges

- Immediate publication on acceptance

- Inclusion in PubMed, CAS, Scopus and Google Scholar

- Research which is freely available for redistribution 